\title{
Effect of roast degree, preparation method, and variety in the sensory and chemical characteristics of coffee (coffea arabica): A mid-infrared spectrum analysis
}

\author{
Yeison Fernando Barrios-Rodríguez ${ }^{1 *} \mathbb{B}$, Gisela-Andrea Córdoba ${ }^{2} \mathbb{B}$, Andrés Felipe Bahamón-Monje ${ }^{\mathbb{B}}$, Nelson Gutiérrez-Guzmán ${ }^{\mathbb{B}}$
}

${ }^{1}$ Pontificia Universidad Católica de Chile, Department of Chemical and Bioprocess Engineering, School of Engineering, Santiago, Santiago, Chile 2Universidad Surcolombiana - Facultad de ingeniería, Centro Surcolombiano de Investigación en café "CESURCAFE". Neiva, Huila, Colombia Corresponding autor: yfbarrios@uc.cl; u20132122474@usco.edu.co; andres.bahamon.coffee@gmail.com; ngutierrezg@usco.edu.co Received in September 6, 2021 and approved in November 4, 2021

\begin{abstract}
In coffee beverages, there are several factors that affect the final compounds and generate sensory variations. This study evaluated the effect of five preparation methods, three roast degrees, and three different varieties (coffea arabica) on the physicochemical compounds of coffee (coffea arabica) before and after preparation by using information obtained from the mid-infrared spectrum. The effect on some sensory attributes was assessed by means of a panel of 54 habitual coffee consumers. Spectrum data were processed using hierarchical clustering and principal component analysis (PCA), while a mixed general linear model was applied for sensory analysis. The results showed that each factor behaves independently, showing a significant effect ( $p<$ 0.05 ) on a greater number of attributes. The preparation method and the roast degree are attributed to the changes generated in the chemical characteristics of coffee during these processes. Through the analysis of the infrared spectrum (IR) by hierarchical cluster, it was found that, before the preparation of the coffee drinks, the samples are grouped by roast degree, regardless of the type of variety. Spectrum analysis by PCA after brewing indicated that there is a greater effect of the roast degree and variety of coffee (coffea arabica) on the chemical markers of the IR spectra. Finally, wavelengths 1,800 , $1,740,1,650,1,550,1,480,1,150$, and $710 \mathrm{~cm}^{-1}$, which had a greater contribution in the PCA and allowed the evaluated samples to be grouped differently, are those related to non-volatile compounds such as caffeine, chlorogenic acids, and trigonelline, which provide sensory characteristics to the final drink.
\end{abstract}

Key words: Coffee beverage; Food processing; FTIR; Sensory evaluation.

\section{INTRODUCTION}

Coffee is one of the most commercialized foods worldwide, according to the international coffee organization (ICO). Worldwide coffee exportations amounted to 10.3 million bags in December 2019, compared to 10.17 million in December 2018. The countries of the European Union and the United States are the main coffee importers worldwide with $64.5 \%$ and $11.3 \%$, respectively of the total $60-\mathrm{kg}$ sacks imported in 2018 (International Coffee Organization - ICO, 2019). Colombia is the second largest producer of Arabica coffee in the world, and its harvest in 2019/20 is forecast to reach 14.1 million bags, $1.7 \%$ more than in 2018/19 according to the report for ICO in 2019.

One of the main attractions of coffee are its unique characteristics of flavor and aromas. However, these sensory characteristics greatly throughout due to the influences of variety, geographic location, unique climates, different agricultural practices, variations applied in the processing method, and type of roasted (Sunarharum; Williams; Smyth, 2014). Some of these factors are more complex to study than others, for example, flavor development during roasted of coffee is highly complex. In the roasting hundreds of chemical reactions take place simultaneously such as the Maillard and Strecker reaction, degradation of proteins and polysaccharides (Farah et al., 2006). Conditions as roast degree and time/temperature determine the formation of compounds, that are responsible for the desirable flavors and aromas on coffee (Masi et al., 2013). On the other hand, the coffee preparation methods are quite variable in the technical conditions applied, such as the coffee/water ratio, the temperature and pressure, which contribute to the chemical compositions of the beverage (Pérez et al., 2010) and generate extracted coffees with different sensory profiles (Gloes et al., 2013).

To study the effect of various factors on the properties of the coffee beverage, different analytical techniques have been applied, such as solid phase microextraction gas chromatography together with mass spectrometry (SPMEGC / MS) (Caporaso et al., 2014; Gloes et al., 2013), proton transfer reaction mass spectrometry (PTR-MS) (Bodner et al., 2019), as well as mass-associated high-efficiency liquid chromatography (HPLC-.MS). These techniques generate good results; however, require the preparation of samples, time and reagents that in some cases are polluting for the environment. The mid-infrared spectroscopy technique has shown that together with the multivariate analysis of the data it can be used to examine and evaluate the chemical composition of coffee (Lyman et al., 2003; Craig et al., 2018; Craig; Franca; Oliveira, 2011; Garrigues et al., 2000). Additionally, has the advantage that it does not require reagents or sample preparation, and the results are obtained in a short period of time. 
According to what has been described previously, the aim of this work was to assess the effect of the preparation method, variety and roast degree on the chemical composition before and after the preparation of the coffee beverage using the mid-infrared spectrum. In addition, the effect on 10 sensory attributes were evaluated with a panel of habitual coffee consumers.

\section{MATERIAL AND METHODS}

\subsection{Roasted coffee}

Three coffee samples (Coffea arabica) were selected: Caturra, Colombia and blend coffee (70\% Castillo and 30\% Caturra) varities, whose sensory profile defined them as special coffees in accordance with the established by the protocol of the SCA (Specialty Coffee Association). For the roasting process, the green coffee beans were previously homogenized in sieves with diameters of 5,6 and $6.7 \mathrm{~mm}$. Later they were taken to a Quantik roaster (TC-150A R / G), with an initial temperature of $180 \pm 5^{\circ} \mathrm{C}$, and an outlet temperature of $160 \pm$ $5^{\circ} \mathrm{C}$. Procedures are performed in triplicate for each variety.

The roast degree was determined according to the ranges established by Wei and Tanokura, (2014), in luminosity values * L of the CieL*a*b coordinate, using the Minolta CR 410 colorimeter (Konica Minolta Sensing Inc., Osaka, Japan), as follows: light $(\mathrm{L}>24)$, medium $(21>\mathrm{L}<24)$ and dark $(\mathrm{L}<21)$. Then the beans were ground in a Gindmaster mill (810S Black ETL SlimLin) for 15 seconds with a medium ground size.

\subsection{Sensory acceptance analysis}

The samples were evaluated by fifty-four judges, frequent consumers of special coffee and with experience in the sensory analysis of coffee. Nine sessions were held, each one with a panel of six tasters. The attributes evaluated were: aroma, color, sweetness, residual flavor, flavor, body, acidity and general impression, rated on a 9-point scale from "Not acceptable” (0) to “Acceptable” (9). The samples preparation was carried out randomly and the containers were labeled with three-digit random codes that corresponded to each one of the samples under study.

The amount of coffee samples to be evaluated were determined as recommended by the ISO 6668:2008 reference, which establishes a ratio of $7 \mathrm{~g}$ of coffee per $100 \mathrm{~mL}$ of water (NTC, 2002). The grinding of the samples was carried out one day before the preparation, in order to preserve the freshness and volatile compounds of the coffee samples. The level of grinding in this study was the same, to focus only on the coffee beverage preparation method, as recommended by Sanchez and Chambers, (2015). Carbon filtered water was used to guarantee neutrality, the absence of unwanted odors or flavors in the water that reaches the beverage (Lawless et al., 2005;
Hoehl et al., 2010). The samples preparation was carried out using the following preparation methods:

- Chemex: $21 \mathrm{~g}$ of ground coffee were used and $300 \mathrm{~mL}$ of water was poured at $90{ }^{\circ} \mathrm{C}$. During the process, a soft, slow and constant drizzle of water was carried out in a circular manner in the chemex, in an average preparation time of 4 minutes.

- Dripper/V60: $21 \mathrm{~g}$ of ground coffee were used, followed by $300 \mathrm{~mL}$ of water at $90{ }^{\circ} \mathrm{C}$, gently sprayed until the coffee was wet without stopping dripping, then circularly from the inside, moving outwards and waiting 45 seconds. Finally, the dumping in circular motion was resumed until the total amount of water was reached, with an average preparation time of 3.5 minutes.

- $\quad$ Aeropress (SKU: AE-002): The plunger was located inside the base and $10.5 \mathrm{~g}$ of freshly ground coffee were added; subsequently, a previously soaked paper filter was placed in the filter holder. Then $150 \mathrm{~mL}$ of water were poured at $90{ }^{\circ} \mathrm{C}$ in two stages, stirring in the middle and at the end. It was left to rest for 45 seconds, and the filter holder was placed on the AeroPress. The coffee maker was set to exert a constant force until all the coffee was extracted. The average preparation time for the coffee drink was 1.5 minutes

- $\quad$-Home elements conventional electric coffee maker (Mhe7031): $21 \mathrm{~g}$ of ground coffee were added to the machine's filters, as well as $300 \mathrm{~mL}$ of water at $90{ }^{\circ} \mathrm{C}$, with an average preparation time of 1.3 minutes.

- Moka pot: Water was added to the safety valve at the bottom of the Italian coffee machine. The filter was put in, and $21 \mathrm{~g}$ of coffee was added. The water was heated to boiling point, with a preparation time of 1.5 minutes.

\subsection{Mid-infrared spectrum analysis}

The coffee samples were analyzed in the IR spectroscopy before extraction (ground coffee beans) and after carrying out the extraction of the coffee beverage (spend coffee grounds). In order to obtain a better interpretation of the coffee beans spectra, the samples were brought to a same value of water activity $(0.418 \pm 0.034)$, for which it was necessary to previously dry the samples at a temperature of $70{ }^{\circ} \mathrm{C}$ during 14 hours and then left in a desiccator for 48 hours. Water activity measurements were made on a steam sorption analyzer, at $15^{\circ} \mathrm{C}$. (VSA Aqualab Decagon Device, Inc. Pullman, WA).

The IR spectrum were performed on ATR-FTIR Cary 630 (Agilent spectrophotometer, Santa Clara, USA), in a dry atmosphere and at room temperature $\left(20 \pm 0.5^{\circ} \mathrm{C}\right)$, using a horizontal ATR sampling accessory (Diamond ATR). Approximately $500 \mathrm{mg}$ of ground coffee were placed in the sampling device and pressed; background material was obtained from fixture readings without any samples. All spectra were recorded within a range of $4000-650 \mathrm{~cm}^{-1}$ with a resolution of $8 \mathrm{~cm}^{-1}$ and 20 scans. 
In total, 45 samples of spend coffee ground and 9 samples of roasted ground coffee were analyzed. Five repetitions were made to each sample, to obtain a total of 270 spectra. The information IR were exported to Excel format for further analysis.

\subsection{Statistical analysis}

The data obtained from the sensory acceptance were subjected to a general analysis and mixed linear models, considering the variety, the toast degree, the coffee preparation methods, the double and triple interactions as defined factors. Results are expressed as the mean \pm standard error of the mean (SEM). Fisher's means were used to estimate the differences between the mean values of the different treatments, with a confidence level 95\% $(\mathrm{p}<0.05)$.

The data obtained from the spectra were subjected to baseline correction and subsequently, normalized. A hierarchical cluster analysis was performed to observe the grouping of the samples according to the roast degree before the preparation of the beverage, using as a criterion a distance equal to $50 \%$ of the maximum distance to trace the reference line. For post-brew analyzes, data obtained from relative absorbance of coffee grounds were subtracted from spectrum absorbance in roasted coffee. Then the data was analyzed by principal component analysis (PCA) to observe the similarities between the extraction methods. For the analysis, the region $1800-650 \mathrm{~cm}^{-1}$ was used, this spectral region was selected because they are rich in information, while the inclusion of other spectral data could interfere with the extraction of useful information. Matrices of size 45x307 were constructed, so that each row corresponded to a sample and each column represented the data of the spectrum in a given wavenumber. The data were scaled, dividing by the standard deviation of each of the variables and were centered by subtracting the mean, in order to decrease the variability of the data. The analysis was carried out using the R Core Team (2020) GPU General Public License.

\section{RESULTS}

\subsection{Sensory acceptance}

The results of the statistical analysis for sensory acceptance are shown in Table 1. The marginal hypothesis tests (SC type III) of the classification model throwed results for each interaction and factor, showing that double and triple interactions do not have a significant effect $(p>0.05)$ in none of the evaluated attributes.

Factors as coffee preparation methods and roasted have an effect on all the evaluated attributes, while the variety significantly affects $(p<0.05)$ the attributes of color, sweetness, aftertaste, body and bitter. Figure 1 shows the averages to: method preparation, roast degree and variety.

The chemex and dripper preparation methods presented the highest average scores for all attributes, while the moka pot preparation method presented the lowest average scores (Figure 1a). The figure 1-b shows the means for each roast degree, independently of the coffee preparation method and the variety. It is evident that coffee with a light roast degree presented statistically significant differences $(p<0.05)$ with medium and dark roast in the attributes of aroma, aftertaste, flavor, body, acidity and scoring. The best scores are observed for the castle variety. The Caturra variety shares similarities with the blend sample in sweetness and astringency attributes; while the Castillo and blend are similar in body and color attributes (Figure 1-c).

\subsection{Description of the mid-infrared spectrum}

Figure 2 shows the IR spectra of roasted coffee of different varieties to three-roast degree, between the wavenumber of $1800-650 \mathrm{~cm}^{-1}$. In this region of the IR spectrum of coffee, functional groups associated with some compounds present in coffee have been reported that contribute to the sensory properties.

Table 1: p-value obtained from marginal hypothesis tests (SC type III) for each of the factors and interactions.

\begin{tabular}{ccccccccc}
\hline & $\mathrm{V}$ & $\mathrm{RD}$ & $\mathrm{PM}$ & $\mathrm{V} / \mathrm{R}$ & $\mathrm{V} / \mathrm{Pm}$ & $\mathrm{R} / \mathrm{Pm}$ & $\mathrm{V} / \mathrm{R} / \mathrm{Pm}$ \\
\hline Aroma & 0.089 & 0.001 & 0.001 & 0.105 & 0.493 & 0.677 & 0.117 \\
Color & 0.035 & $<0.0001$ & $<0.0001$ & 0.116 & 0.095 & 0.760 & 0.891 \\
Sweetness & 0.001 & 0.013 & 0.001 & 0.680 & 0.906 & 0.743 & 0.789 \\
Aftertaste & 0.016 & 0.006 & $<0.001$ & 0.054 & 0.334 & 0.446 & 0.196 & 0.833 \\
Flavor & 0.130 & 0.005 & $<0.0001$ & 0.118 & 0.330 & 0.150 & 0.761 & 0.903 \\
Body & 0.011 & $<0.0001$ & $<0.0001$ & 0.714 & 0.314 & 0.588 & 0.835 \\
Acidity & 0.103 & 0.001 & $<0.001$ & 0.897 & 0.733 & 0.748 & 0.580 \\
Bitter & 0.018 & 0.069 & 0.035 & 0.613 & 0.368 & 0.574 & 0.554 \\
Scoring & 0.171 & $<0.001$ & $<0.0001$ & 0.119 & & &
\end{tabular}

V: Variety; RD: Roast degree; PM: Preparation method. 
(a)

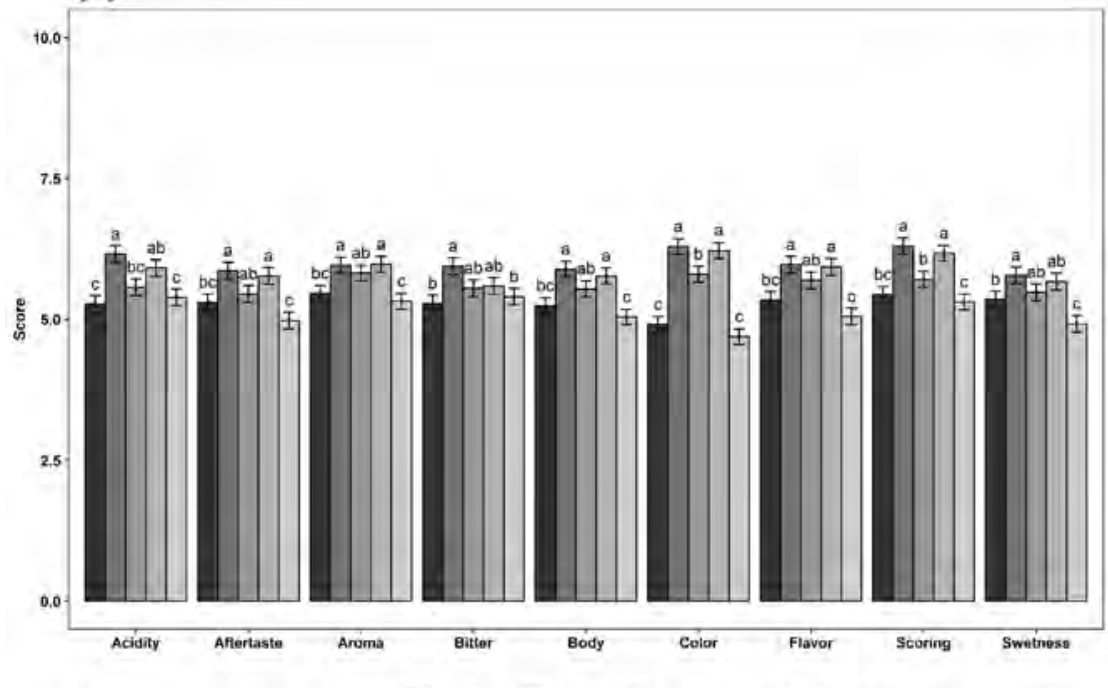

(b)

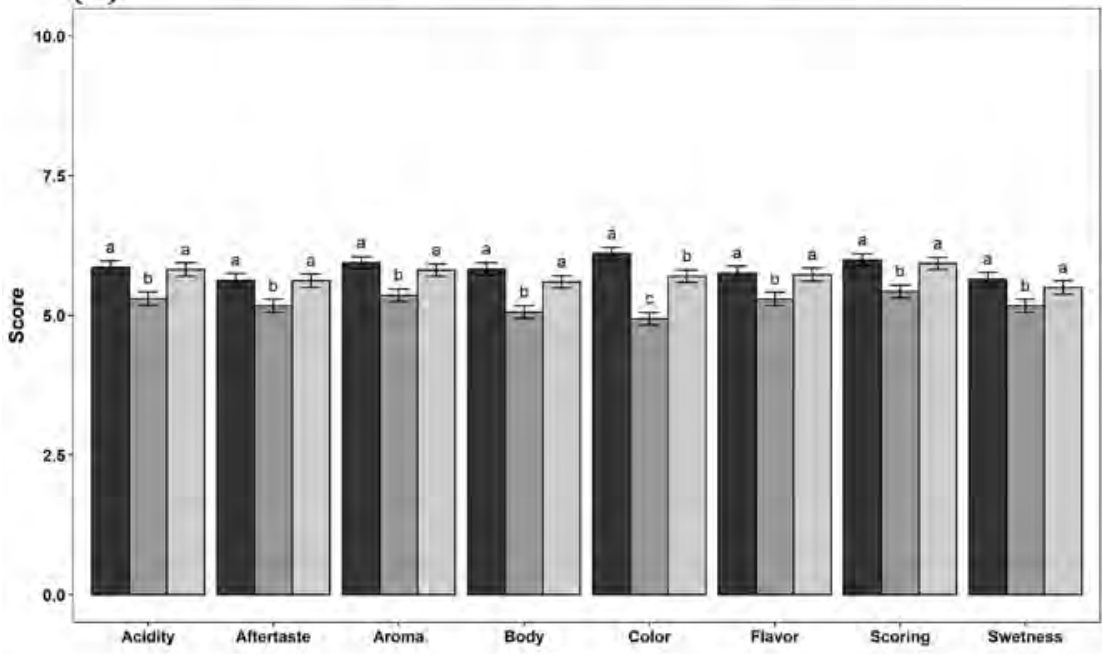

(c)

Roast degree $\square$ Dark $\square$ ignt $\square$ mesium

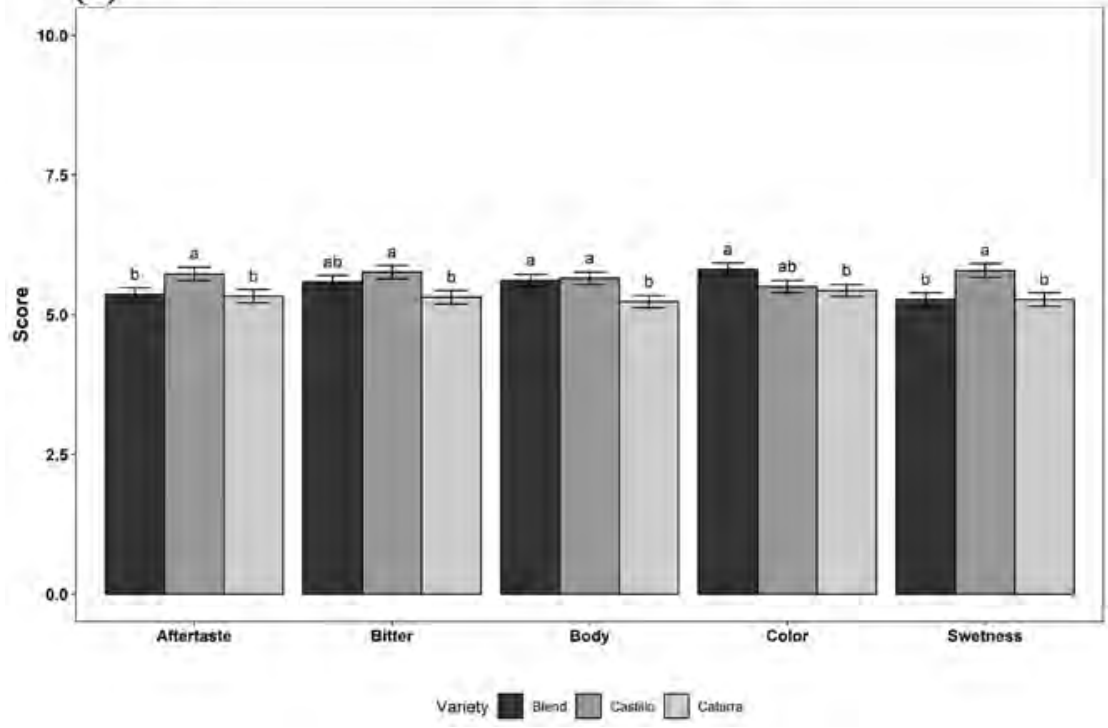

Figure 1: Averages and standard error for sensory attributes according to: (A) Preparation method, (B) Roasted and (C) Variety. Different letters in the horizontal direction, in the same attribute, indicate statistically significant differences $(p<0.05)$. 


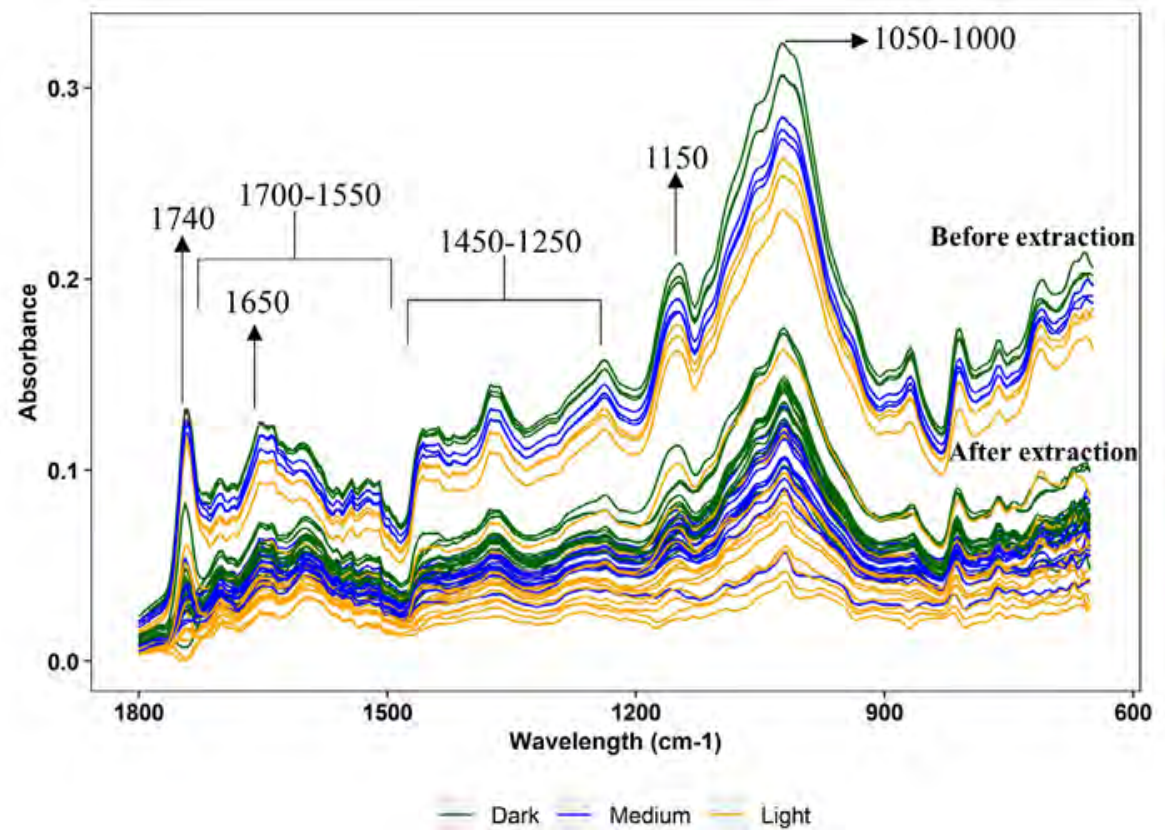

Figure 2: IR spectra normalized obtained for each of the samples between the wavenumber of $1800-650 \mathrm{~cm}^{-1}$ for ground coffee to different roast degree (upper) and spend coffee grounds after the preparation of the beverage (down).

Once the coffee beverage has been extracted, the absorbance regions identified in roasted coffee decreases its absorbance (Figure 2). Depending on the coffee preparation methods its relative absorbance value decreases differently. On the other hand, Figure 2 shows that there were greater differences between the absorbance values at the different roast degree than between varieties with the same roast degree. However, from the visual analysis it is not possible to distinguish some type of grouping by any of the evaluated factors, therefore a multivariate statistical analysis of the IR spectra data is required to better explore the observed differences.

\subsection{Mid-infrared spectrum analysis}

A hierarchical cluster analysis was performed with the information obtained in the IR spectra between $1800-650 \mathrm{~cm}^{-1}$ the roasted coffee beans (before the preparation of the coffee beverage). The results show a clear separation by type of roast degree, independently of the variety (Figure 3).

Considering the initial absorbance values in roasted coffee before the preparation of the coffee beverage, minus the absorbance values in the spend coffee grounds after preparation, a tentative data of the possible compounds present in the beverage was obtained, which it was evaluated if there were differences between each extraction method. These were analyzed using PCA (Figure 4) and according to the results of the hierarchical cluster, each roast degree was analyzed separately, in order to show how the different coffee preparation methods and varieties are grouped together at the same roast degree.

A PCA was performed, integrating all the information, to observe if the samples maintain the evidenced grouping by roast degree before the preparation of the beverage. However, the PCA results did not show a specific grouping, but a trend of the samples by roast degree. A new cluster analysis was performed from the first 5 components obtained (Figure 5), to reduce the noise of the information obtained from the spectra. It observed a tendency for the samples to be grouped by the roast degree and the variety, rather than by the method of preparation of these.

\section{DISCUSSION}

\subsection{Sensory acceptance}

The factors (variety, roasted and metho preparation) presented a behavior independently, do not depend on each other. Sanchez and Chambers (2015) also reported that the interaction of the coffee preparation methods and type of coffee do not have a significant incidence on the sensory attributes evaluated by a panel of consumers, so the information obtained from each factor must be analyzed independently.

In this sense, it is showed that a greater acceptance sensory by tasters for blend coffee, than for Caturra and Colombia coffee varieties. Which may be due to the fact that the mixture between arabica coffee varieties potentiates some attributes of the coffee and improves sensory acceptance by the consumer. On the other hand, previously described agrees with the one reported by Donfrancesco, Gutierrez Guzman and Chambers (2019) with samples of special coffee from the same sub-region of Colombia. These authors evidenced that the samples had similar sensory properties, although not exactly the same, differentiating according to their characteristics such as 
caramel, pungent and ashy aroma, or bitter, acidic and astringent flavors (Donfrancesco; Gutierrez Guzman; Chambers, 2019). The little differences detected between coffee samples (Coffea arabica) from the same region may probably be due to the specific and unique combinations of aspects such as geographical area, climatic characteristics, altitude and practices adopted by each farm. Another factor that may influence are maturity of cherries when it is collected, which produces a change in the chemical and sensory characteristics of the roasted coffee beans that have an impact on the sensory acceptance of some attributes (Velásquez et al., 2019).

On the other hand, the light roast degree showed lower sensory acceptance. Other studies have shown that a light roast can prevent the complete development of the aroma and result in a lower concentration (Bodner et al., 2019). The dark roast was mainly related to an increase in attributes such as bitter, astringent and sometimes burned (Giacalone, 2019), and decrease of compounds such as chlorogenic acids, related to attributes such as acidity (Barbosa et al., 2018). However, the results of the sensory analysis showed a greater acceptance for dark roast for acidity attribute, which may be due to what the medium and mediumhigh roast process leads to a more balanced flavor and aroma, often with flavor citrus notes (Bodner et al., 2019).

The differences obtained in the flavor and aroma to different roast degree, attribute due to the fact that in more dark roast degree, compounds that are produced during the roasting process are related to bitter or astringency sensation in the beverage, being classified as a coffee flavor characteristic by consumers (Barbosa et al., 2019; Nebesny; Budryn, 2006). This could also lead to a better result in the scoring attribute, considered as the overall score by the consumer. Finally, in the sweetness attribute, the degrees of light and medium roast presented significant differences with the degree of dark roast, which exhibited a lower acceptance mean. This is consistent with the results evidenced by Bhumiratana, Adhikari and Chambers (2011), who reported a loss of attribute sweetness in samples with higher roasted, possibly due be further carbohydrate breakdown during the dark roasting coffee.

Regarding the differences between the methods may be due to the fact that in the filtration methods (chemex and v60), the coffee beverage is prepared by infusing roasted beans followed by filtration, while moka pot is a method that is classified as a pressurized method, where water pressure is used to obtain the beverage (Fibrianto; Fakhruddin; Wulandari, 2019). The moka pot preparation method generates a stronger coffee, due to the increasing pressure in the system and temperature change in the steam water increase the body intensity and odor, as well as the bitterness of the beverage, which generally leads to less consumer acceptance (Cordoba, 2010). This method presents a relatively higher content of caffeine, feeling a greater astringency in the beverage (Pérez et al 2010). The filtration methods water is used equal to or below the boiling point, with a greater time of contact between the water and the coffee bean. This increases the solubility of the volatile compounds responsible for the flavor present in the coffee, generating a higher intensity of the attributes in the beverage at the time of the sensory analysis (Sanchez; Chamber 2015, Cordoba, 2010). The Aeropress method, which also requires pressure, presented significant differences in the attributes of aroma, aftertaste, flavor and body with the chemex and dripper methods. However, in the Aeropress

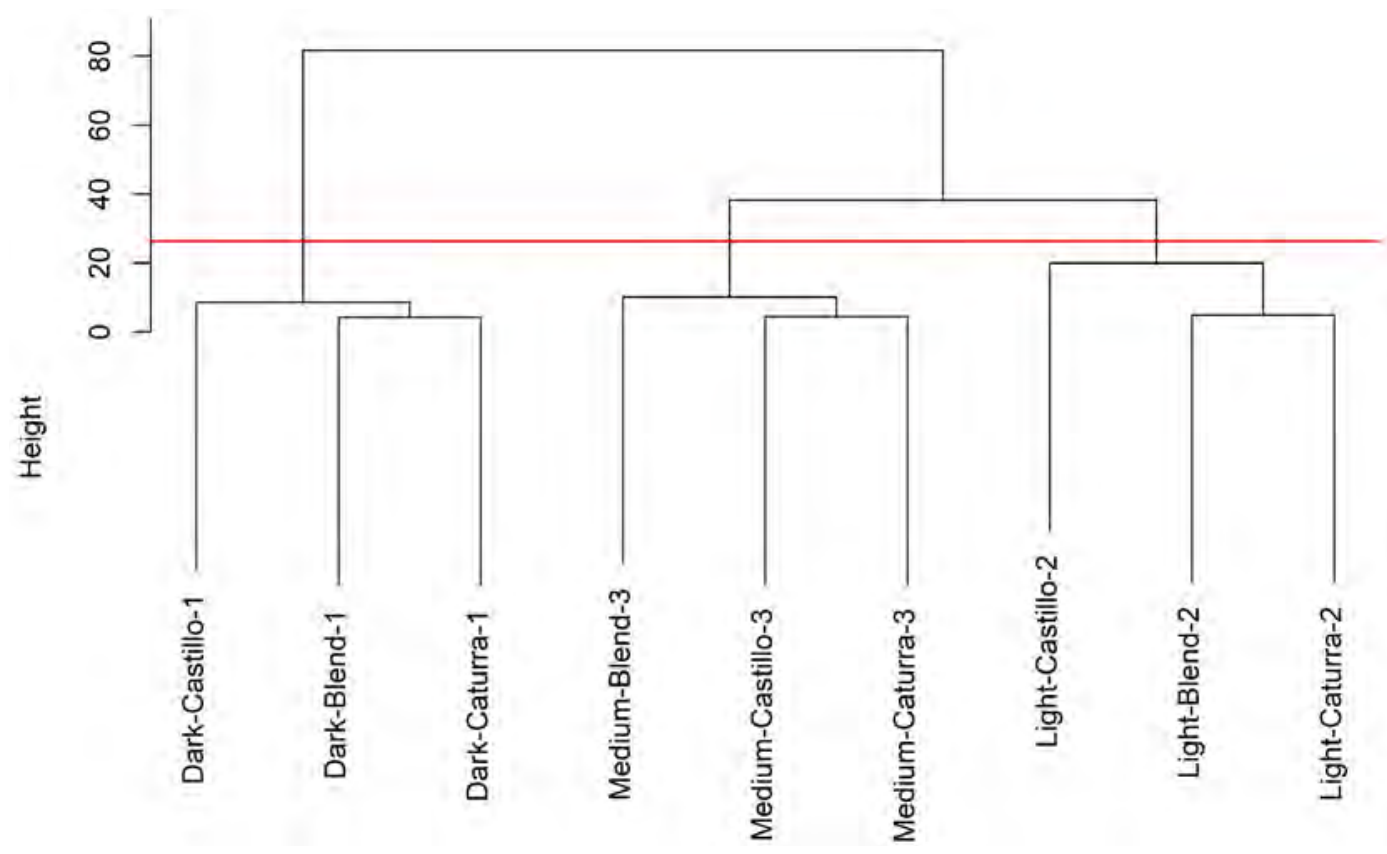

Figure 3: Hierarchical cluster for the data obtained from the IR spectra of roasted coffee to different roast degrees. 
method the preparation time is variable, which can contribute to accentuate particular flavors depending on the time of contact water/coffee. Finally, in the coffee brewing method with coffee maker, the smallest differences were observed with the dripper and chemex methods, presenting more similar average scores.

\subsection{Description of the mid-infrared spectrum}

The sensory results are also explained by the evidenced in the infrared spectrum (Figure 2). The differences in the absorbances observed in the spectrum are associated with the concentration of the different coffee compounds (caffeine, chlorogenic acids, quinic acid, carbohydrates, trigonelline and lipids, among others), which influence sensory acceptance. The caffeine influences perceived strength, body and bitterness of coffee drink (Sunarharum; Williams; Smyth, 2014). As for chlorogenic acids and qunic, their presence contributes to greater astringency and bitterness (Buffo; Cardelli, 2004). The alkaloid presents in coffee, trigonelline, has been related to better aroma perception (Oestreich, 2010) and the presence of polysaccharides such as sucrose and fructose may perceived sweetness flavor in coffee (Sunarharum; Williams; Smyth, 2014). In the spectra vibrations showed in 1750-1600 $\mathrm{cm}^{-1}$ (Figure 2), can be attributed to the axial deformation of $\mathrm{N}-\mathrm{H}$ group in the aromatic ring due to the possible presence of alkaloids such as caffeine (Barrios; Giron; Gutierrez, 2020a, Barrios et al., 2021a; Craig et al., 2018; Wang et al., 2009). It has been observed $\mathrm{O}-\mathrm{H}$ single bond angular deformation, axial $\mathrm{C}-\mathrm{O}$ single bond deformation and the $\mathrm{C}$ - $\mathrm{O}$ - $\mathrm{C}$ single bond single bond ester bond, in the wavenumbers between 1600-1300 $\mathrm{cm}^{-1}$ and $1450-1250 \mathrm{~cm}^{-1}$, associated to trigonelline, quinic acid and chlorogenic acids (Barrios et al., 2021b; Craig et al., 2015; Reis; Franca; Oliveira, 2013). Finally, the pick in the range $1050-1000 \mathrm{~cm}^{-1}$, this may be to axial C-O deformation of the quinic acid. On the other hand, the C-O-C ester bond absorbs in the $1300-1000 \mathrm{~cm}^{1}$ range (Craig et al., 2012). In the region $1065-1020 \mathrm{~cm}^{-1}$ may also be due to the presence of arabinogalactans (Barrios et al., 2021b). In general, differences are observed in all regions of the spectrum according to the roast degree and the preparation method (Figure 2), which indicates that it is possible to establish which treatments are similar in their chemical composition by analyzing the information of the infrared spectrum.

\subsection{Mid-infrared spectrum analysis}

In the cluster analysis for the data of the spectrum in ground coffee before the preparation of beverages (Figure 3), it is evident that, at the same roast degree, the varieties share similarities in their chemical composition. These results are relevant for the subsequent analysis in the spend coffee ground, it also contributes to understand why there is no interaction between the factors varieties and roast degree in the results of sensory analysis. In the PCA (Figure 4), in the light roast degree the $98.5 \%$ of total variance with the first two components are explained, as evidenced in Figure 4-a. It was observed that the coffee preparation methods are dispersed in a different way, but maintain a tendency of grouping according to the variety. The results obtained for this roast degree show that the methods of preparation with the blend coffee tend to be grouped in opposite quadrants to those obtained with the Caturra and Castillo variety, which are grouped in quadrant IV and III, respectively. At a medium roast degree, $94.1 \%$ of the total variance can be explained with the first two components. As seen in Figure 4-b, the distribution is very different from that obtained at a light roast degree. On this occasion, the methods prepared with the Caturra variety are grouped in an opposite sector to the Castillo and blend varieties, which are located mainly in quadrant III. Finally, in roast dark it was possible to explain $95.8 \%$ of the variance (Figure 4-c). The grouping trend evidenced in the medium roast, is also observed at this roast degree where the Castillo and blend varieties are located in the upper part of PC1, while the Caturra variety is distributed in quadrants III and IV. The above show the beverages that are most similar among their chemical composition are those obtained from the blend coffee and the Colombia variety, which is best evidenced at the highest roast degree. This may be due to the effect that the preparation method has on the extraction of these compounds, having an impact on the sensory attributes described above (Sanchez; Chamber 2015; Cordoba, 2010).

On the other hand, the score loads of the variables obtained from the PCA by roast degree, showed that the wavenumber that have a greater contribution in PC1 in the three roast degrees are: 1800, 1740, 1650, 1550, 1480, 1150 and $710 \mathrm{~cm}^{-1}$. They stand out the one that represent the greatest weight in PC1, because this component allows to group better the different samples. These wavenumbers that represent a greater contribution to PC1, as already described above, are associated with certain compounds of interest such as caffeine, chlorogenic acids, lipids, trigonelline and carbohydrates (Barrios et al., 2020; Barrios; Salas; Giron, 2020; Barrios et al., 2021; Craig et al., 2018; Craig; Franca; Oliveira, 2011a, 2011b; Craig et al., 2015; Reis et al., 2013; Wang et al., 2011; Wang et al., 2009; Lyman et al., 2003; Garrigues et al., 2000). Although the presence of these wavenumber is not only due to these compounds, but to the set of vibrations of functional groups of different compounds. The fact, that they have great incidence for the separation of the samples in the study is relevant, since they provide organoleptic characteristics of interest in coffee beverage and can be associated with the sensory results described above. 

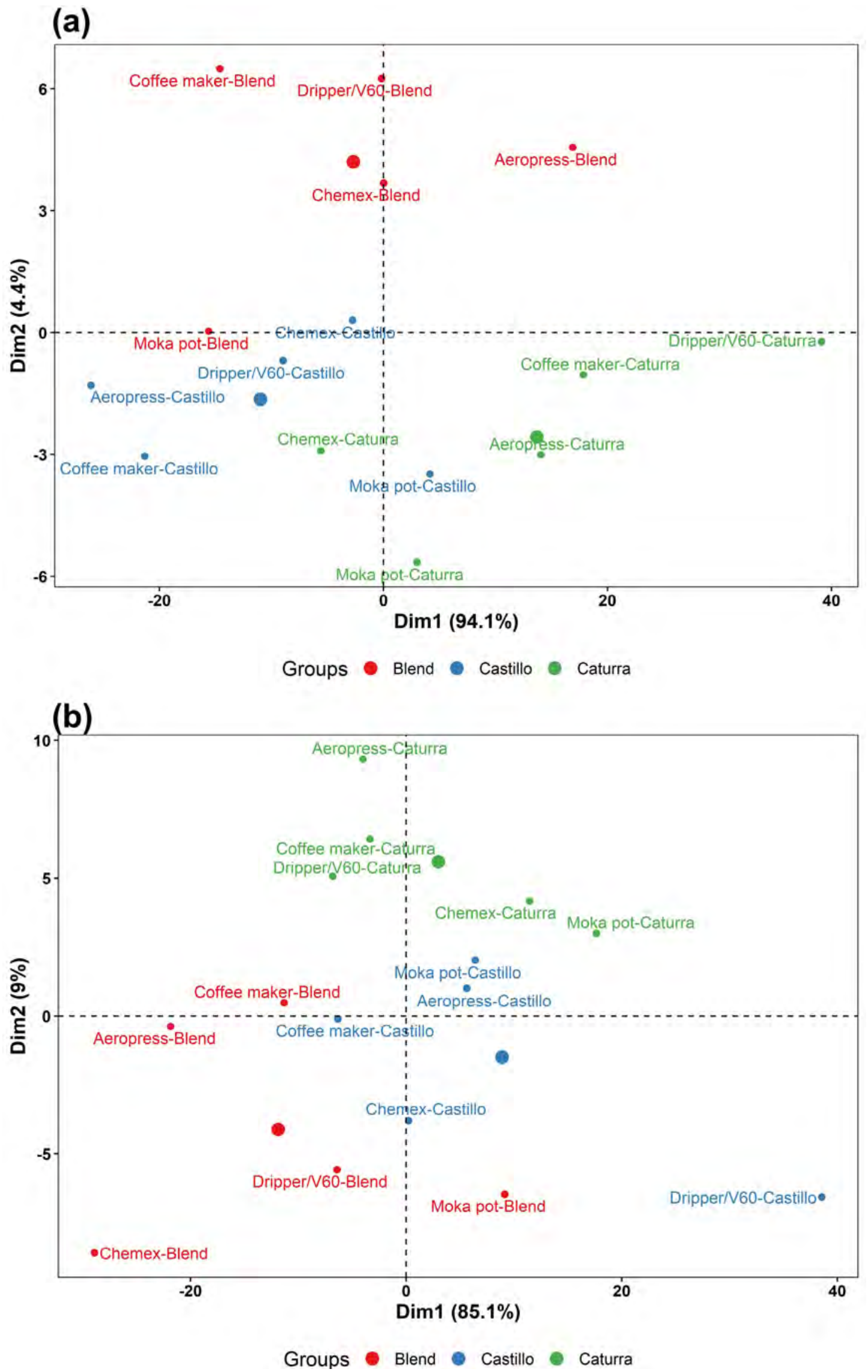

Figure 4: Principal component analysis for spend grounds coffee at each roast degree: (a) light, (b) medium, (c) dark. 


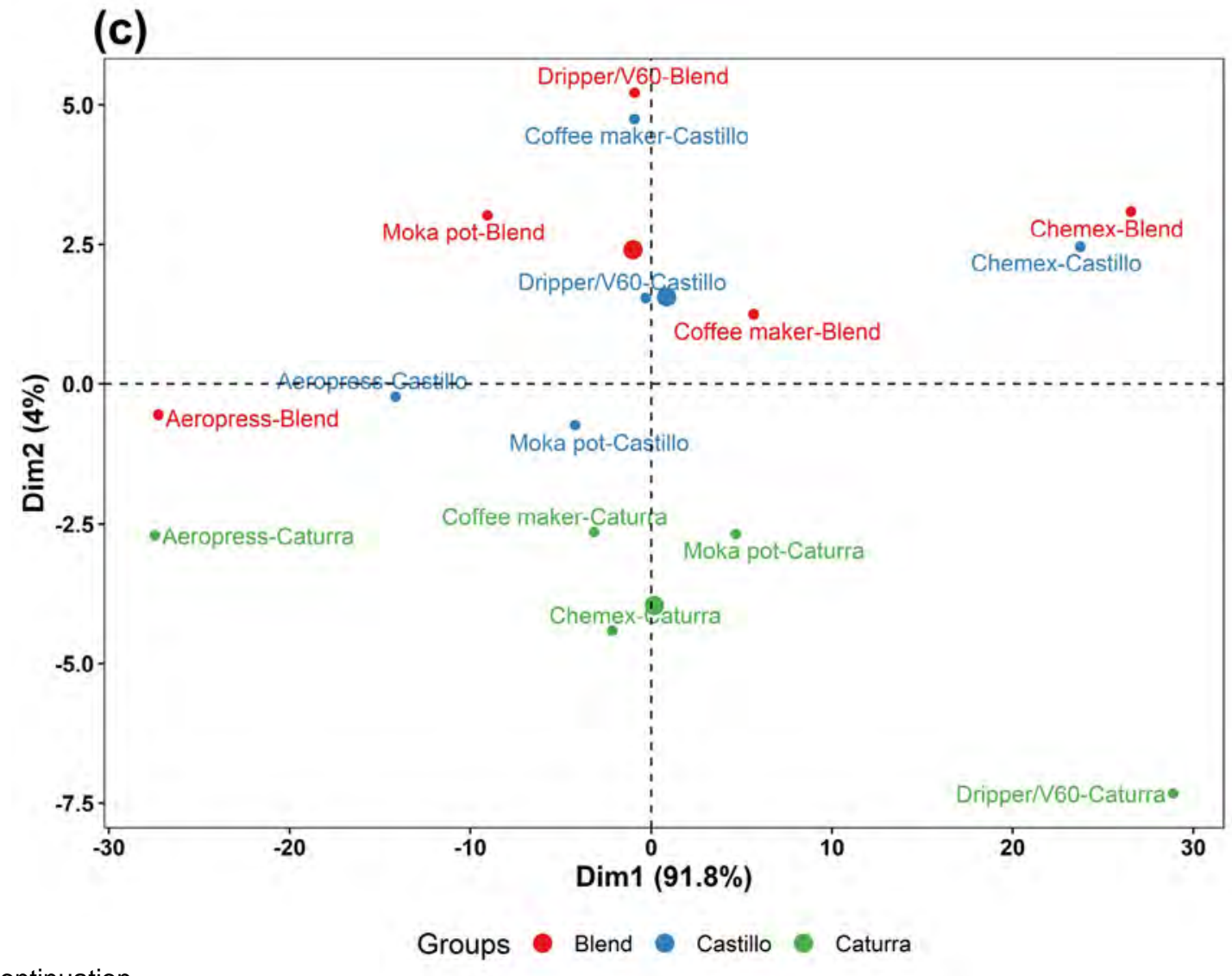

Figure 4: Continuation...

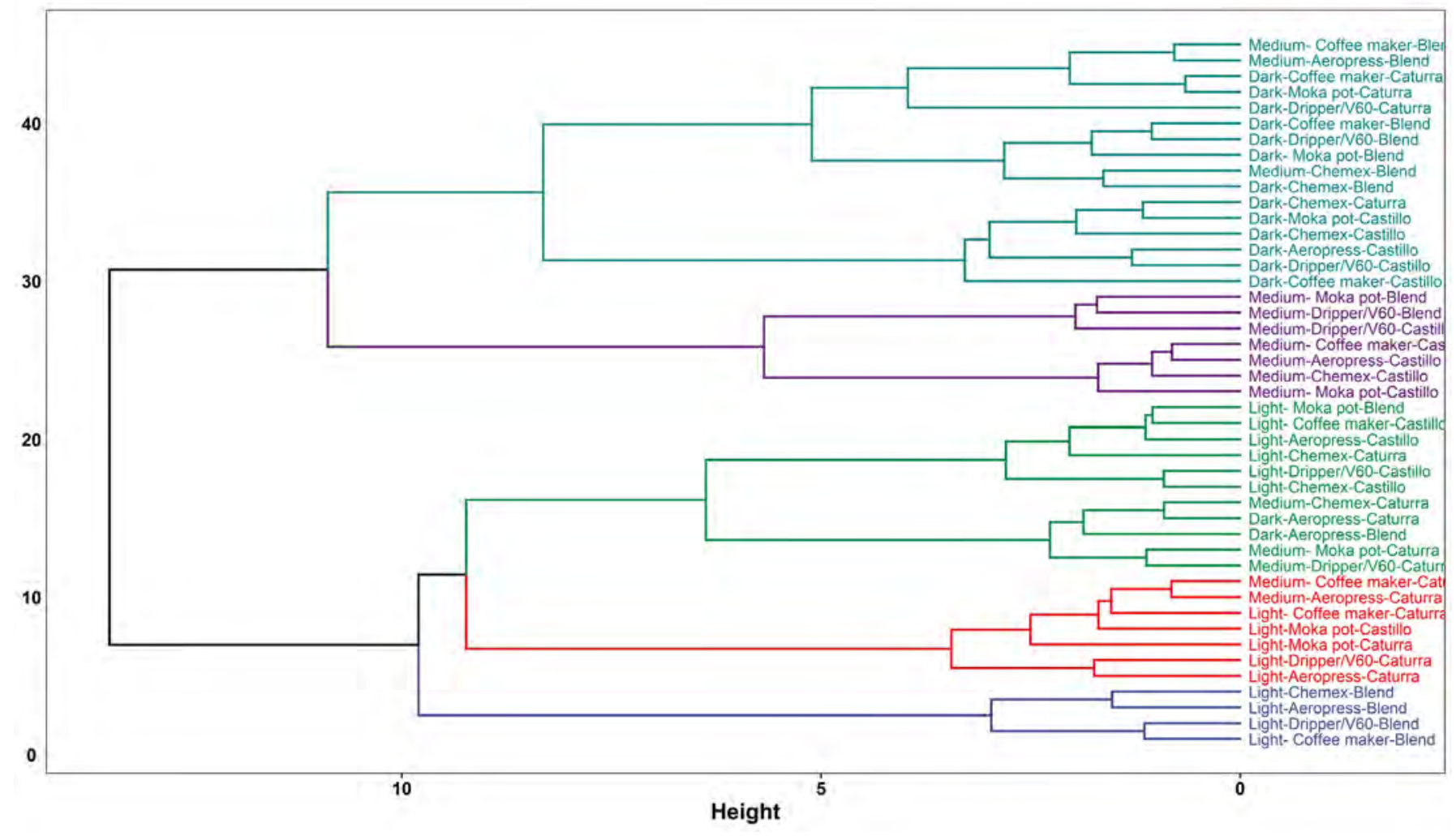

Figure 5: Hierarchical cluster obtained from first 5 components PCA for spend grounds coffee. 
The analysis of the IR spectra indicates that the final compounds in the beverage may be determined by the roast degree and the variety. However, the sensory analysis showed that the coffee preparation method factor affects a larger group of attributes, compared with the other two factors. This means that, although the coffee preparation methods evaluated in the present study contribute to highlight certain attributes which are perceived by the consumer, it does not indicate a difference in concentration of a possible compound in relation to that obtained in other coffee preparation methods.

According to these results, it can be deduced that coffee beverage prepared by any preparation method at light roast, present a discrepancy between their chemical composition with the samples at dark roast, since they are grouped very distant. On the other hand, the medium roast shares a group with the roast light and dark. These may indicate similarities between their chemical characteristics, as is the case of beverage prepared with the moka pot, dripper and chemex method that resemble to the light roast samples, while the coffee maker and Aeropress methods are grouped with a majority vast of samples at dark roast. Likewise, it is highlighted that between samples at the same roast degree the methods are grouped differently, which also agrees with the sensory results where it was evidenced that there are differences in the different levels of the evaluated factors independently.

\section{CONCLUSIONS}

The results of this study indicated that the sensory attributes of the coffee beverage evaluated by a panel of consumers are not affected by the interaction (double or triple) of the factors roast degree, variety and coffee preparation method. The roast degree and coffee preparation method factors showed to have an effect on a larger group of attributes, which was associated with the decrease of interest chemical compounds during these two processes, evidenced in the absorbance levels of the FTIR spectrum for the samples with different roast degree and coffee preparation methods. Among the different preparation methods, it was established that the moka pot method has a big negative impact on the acceptance of the beverage by consumers, while the Chemex and dripper methods have a great acceptance at the sensory evaluation, which was considerate for subsequent analyzes where the brewing method should be involved as a factor for sensory acceptance of coffee samples.

The differences found due to the type of variety in some sensory attributes were attributed to accredited the different chemical composition of the evaluated coffee varieties, which could be evidenced in the statistical analysis of the chemical composition obtained from the
IR spectra, classifying the samples mainly by variety and roast degree. In the same way, the analysis of the IR spectra allowed to deduce that after the extraction of the beverage, a greater effect is evidenced by the roast degree and the variety in the content of chemical composition, than by the coffee preparation method. Finally, it is highlighted that the wavenumber with the greatest contribution to the grouping of the evaluated samples are: 1800, 1740, 1650, 1550, 1480, 1150 y $710 \mathrm{~cm}^{-1}$; they were related to non-volatile compounds in coffee that provide sensory characteristics to the beverage.

\section{DECLARATION OF COMPETING INTEREST}

The authors declare no competing financial interest.

\section{AUTHOR CONTRIBUTIONS}

Y.F.B.R: Writing, methodology, original draft and editing. G.A.C: Writing and performed the experiment. A.F.B.M: methodology and editing. N.G.G: Supervision and revision of the final document.

\section{REFERENCES}

BARBOSA, M. S. G. de. et al. Dynamics of sensory perceptions in arabica coffee brews with different roasted degrees. Journal of Culinary Science and Technology, 17(5):453-464, 2019.

BARRIOS, Y.; GIRON, J.; GUTIERREZ, N. Effect of the postharvest processing method on the biochemical composition and sensory analysis of arabica coffee. Engenharia Agrícola, 40(2): 177-183, 2020.

BARRIOS, Y.; SALAS K.; GIRON, J. Comparison of sensory attributes and chemical markers of the infrared spectrum between defective and non-defective Colombian coffee samples. Coffee Science, 15:e151659, 2020.

BARRIOS, Y. et al. Infrared spectroscopy coupled with chemometrics in coffee post-harvest processes as complement to the sensory analysis. LWT - Food Science and Technology, 145:111304, 2021a.

BARRIOS, Y. et al. ATR-FTIR for characterizing and differentiating dried and ground coffee cherry pulp of different varieties (Coffea Arabica L.). Engenharia Agrícola, 41(1):70-77, 2021b.

BHUMIRATANA, N.; ADHIKARI, K.; CHAMBERS, E. Evolution of sensory aroma attributes from coffee beans to brewed coffee. LWT - Food Science and Technology, 44(10):1185-1191, 2011. 
BODNER, M. et al. Effect of harvesting altitude, fermentation time and roasted degree on the aroma released by coffee powder monitored by proton transfer reaction mass spectrometry. European Food Research and Technology, 145(7):14991506, 2019.

BUFFO, R. A.; CARDELLI, C. Coffee flavour: An overview. Flavour and Fragrance Journal, 19(2):99-104, 2004.

CAPORASO, N. et al. Neapolitan coffee brew chemical analysis in comparison to espresso, moka and American brews. Food Research International, 61:151-160, 2014.

CORDOBA, N. et al. Coffee extraction: A review of parameters and their influence on the physicochemical characteristics and flavour of coffee brews. Trends in Food Science and Technology, 96:45-60, 2010.

CRAIG, A. ET AL. Mid infrared spectroscopy and chemometrics as tools for the classification of roasted coffees by cup quality. Food Chemistry, 145:1051-1061, 2018.

CRAIG, A. P.; FRANCA, A. S.; OLIVEIRA, L. S. Evaluation of the potential of FTIR and chemometrics for separation between defective and non-defective coffees. Food Chemistry, 131(3):1368-1374, 2011a.

CRAIG, A. et al. Fourier transform infrared spectroscopy and near infrared spectroscopy for the quantification of defects in roasted coffees. Talanta, 134:379-386, 2015.

CRAIG, A. et al. Evaluation of the potential of FTIR and chemometrics for separation between defective and nondefective coffees. Food Chemistry, 132(2):1368-1374, 2012.

DONFRANCESCO, B.; GUTIERREZ GUZMAN, N.; CHAMBERS, E. Similarities and differences in sensory properties of high-quality Arabica coffee in a small region of Colombia. Food Research International, 116:645-651, 2019.

FARAH, A. et al. Correlation between cup quality and chemical attributes of Brazilian coffee. Food Chemistry, 98(1):373-380, 2006.

FIBRIANTO, K.; FAKHRUDDIN, M. H.; WULANDARI, E. S. Effect of mokapot brewing temperature on sensory profiling of dampit and tulungagung ijo coffee. IOP Conference Series: Earth and Environmental Science, 130(1):1-6, 2019.

GARRIGUES, J. et al. Fourier transform infrared determination of caffeine in roasted coffee samples. Fresenius' Journal of Analytical Chemistry, 366(3):319-311, 2000.
GIACALONE, D. et al. Common roasted defects in coffee: Aroma composition, sensory characterization and consumer perception. Food Quality and Preference, 71:463-474, 2019.

GLOESS, A. et al. Comparison of nine common coffee extraction methods: Instrumental and sensory analysis. European Food Research and Technology, 136(4):607617, 2013.

HOEHL, K.; SCHOENBERGER, G. U.; BUSCHSTOCKFISCH, M. Water quality and tastes sensitivity for basic tastes and metallic sensation. Food Quality and Preference, 11(1):243-249, 2010.

INTERNATIONAL COFFEE ORGANIZATION - ICO. Coffee market prices continued to climb in december. 2019. Available in: $<$ http://www.ico.org/documents/ cy2019-20/cmr-1219-e.pdf>. Access in: March, 20, 2021.

LAWLESS, H. et al. Metallic taste from electrical and chemical stimulation. Chemical Senses, 30(3):185-194, 2005.

LYMAN, D. et al. FTIR-ATR analysis of brewed coffee: Effect of roasted conditions. Journal of Agricultural and Food Chemistry, 51(11):3168-3171, 2003.

MASI, C. et al. Sensory properties of under-roasted coffee beverages. Journal of Food Science, 78(8):1290-300, 2013.

NEBESNY, E.; BUDRYN, G. Evaluation of sensory attributes of coffee brews from robusta coffee roasted under different conditions. European Food Research and Technology, 114(1):159-165, 2006.

NORMA TÉCNICA COLOMBIANA. NTC 3566. Café verde. preparación de muestras para uso en análisis sensorial. 2002.

OESTREICH, S. Chemistry of coffee. Comprehensive Natural Products II: Chemistry and Biology, 3:10851117, 2010.

PÉREZ, M. et al. Influence of brewing method and acidity regulators on the antioxidant capacity of coffee brews. Journal of Agricultural and Food Chemistry, 58(5):1958-1965, 2010.

R CORE TEAM. R: A Language and Environment for Statistical Computing. R Foundation for Statistical Computing. 2020. Available in: $<$ https://www.R-project. org/>. Access in: March, 20, 2021.

REIS, N.; FRANCA, A. S.; OLIVEIRA, L. S. Quantitative evaluation of multiple adulterants in roasted coffee by diffuse reflectance infrared fourier transform spectroscopy (DRIFTS) and chemometrics. Talanta, 115:563-568, 2013. 
SANCHEZ, K.; CHAMBERS, E. How does product preparation affect sensory properties? An example with coffee. Journal of Sensory Studies, 30(6):499-511, 2015.

SUNARHARUM, W. B.; WILLIAMS, D. J.; SMYTH, H. E. Complexity of coffee flavor: A compositional and sensory perspective. Food Research International, 61:315-315, 2014.

VELÁSQUEZ, S. et al. Volatile and sensory characterization of roast coffees - Effects of cherry maturity. Food Chemistry, 174:137-145, 2019.
WANG, J. et al. Fourier transform infrared spectroscopy for kona coffee authentication. Journal of Food Science, 74(5):385:391, 2009.

WANG, N.; FU, Y.; LIM, L. Feasibility study on chemometric discrimination of roasted. j. Journal of Agricultural and Food Chemistry, 59:3110-3116, 2011.

WEI, F.; TANOKURA, M. chemical changes in the components of coffee beans during roasted. In: Coffee in Health and Disease Prevention. Academic Press, p.8391. Chapter 10. 2014. 